\title{
A Mass in the Stomach Observed after Endoscopic Ultrasonography-guided Fine-needle Aspiration for Pancreatic Cancer
}

\author{
Gwang Ha Kim ${ }^{1,2}$
}

Department of Internal Medicine, Pusan National University School of Medicine ${ }^{1}$, Biomedical Research Institute, Pusan National University Hospital ${ }^{2}$, Busan, Korea

Question: A 68-year-old man underwent EUS-guided fine-needle aspiration (FNA) for a $6.5 \mathrm{~cm}$-sized pancreatic body/tail mass (Fig. 1). EUS-FNA with 4 passes using a $22 \mathrm{G}$ needle was conducted through the upper body of the stomach, and there was no abnormal lesion except minor bleeding in the FNA site (Fig. 2). The diagnosis of pancreatic cancer was confirmed, and the 8th American Joint Committee on Cancer stage was T4N0M0 due to invasion into the celiac artery. Therefore, the patient was administered intravenous chemotherapy (gemcitabine+cisplatin) and remained stable for 6 months. On follow-up upper gastrointestinal endoscopy, a subepithelial tumorlike lesion with central ulceration was detected at the posterior wall of the upper body of the stomach (Fig. 3A). On EUS, the lesion was mainly located in the submucosal layer of the stomach without conglutination to the pancreatic mass (Fig. 3B).

What is the most likely diagnosis?

Answer: The endoscopic biopsy specimen obtained from the subepithelial tumor-like lesion revealed adenocarcinoma, pathologically identical to that of primary pancreatic cancer (Fig. 4). Therefore, needle tract seeding of pancreatic cancer into the stomach was diagnosed.

EUS-FNA is a useful and relatively safe diagnostic mo-

Received: June 26, 2019 Revised: July 19, 2019 Accepted: July 21, 2019

Corresponding author: Gwang Ha Kim

Department of Internal Medicine, Pusan National University School of Medicine, and Biomedical Research Institute, Pusan National University Hospital, 179 Gudeok-ro, Seo-gu, Busan 49241, Korea

Tel: +82-51-240-7869, Fax: +82-51-244-8180, E-mail: doc0224@pusan.ac.kr

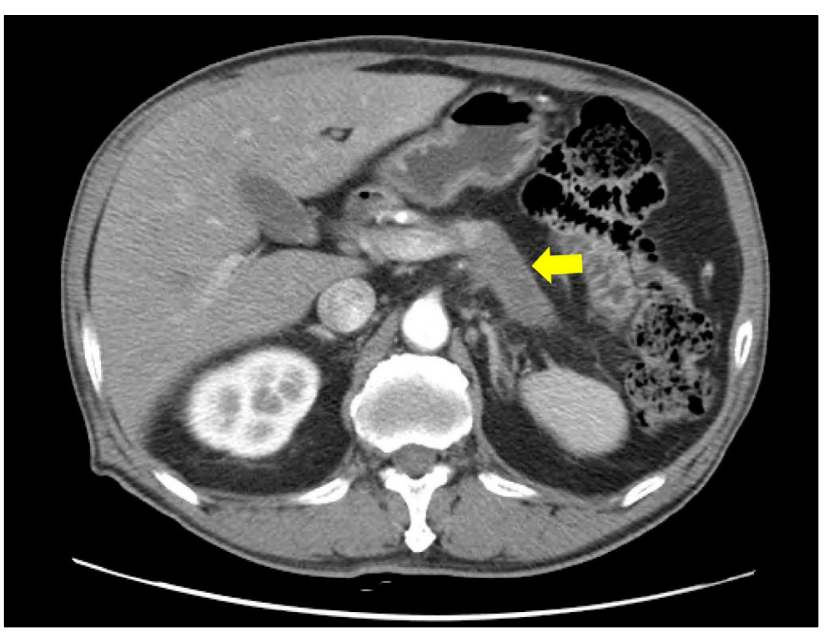

Fig. 1. Abdominal CT reveals a hypovascular mass in the pancreas body and tail (arrow).

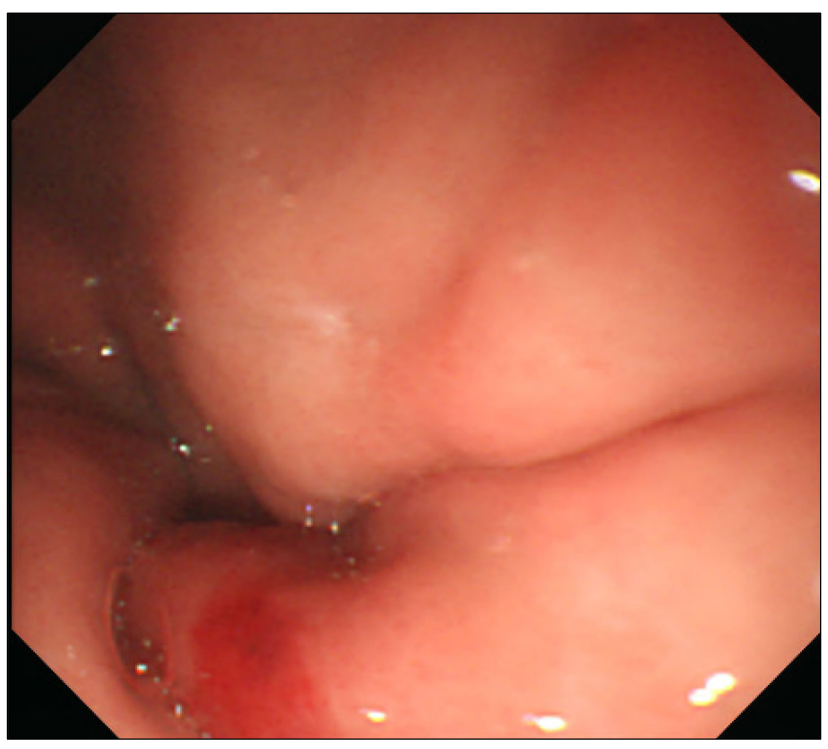

Fig. 2. After EUS-guided fine-needle aspiration, no abnormal lesions were observed except for minor bleeding in the upper body of the stomach.

Copyright $\odot 2019$ Korean College of Helicobacter and Upper Gastrointestinal Research

@ The Korean Journal of Helicobacter and Upper Gastrointestinal Research is an Open-Access Journal. All articles are distributed under the terms of the Creative Commons Attribution Non-Commercial License (http:// creativecommons.org/licenses/by-nc/4.0) which permits unrestricted non-commercial use, distribution, and reproduction in any medium, provided the original work is properly cited. 

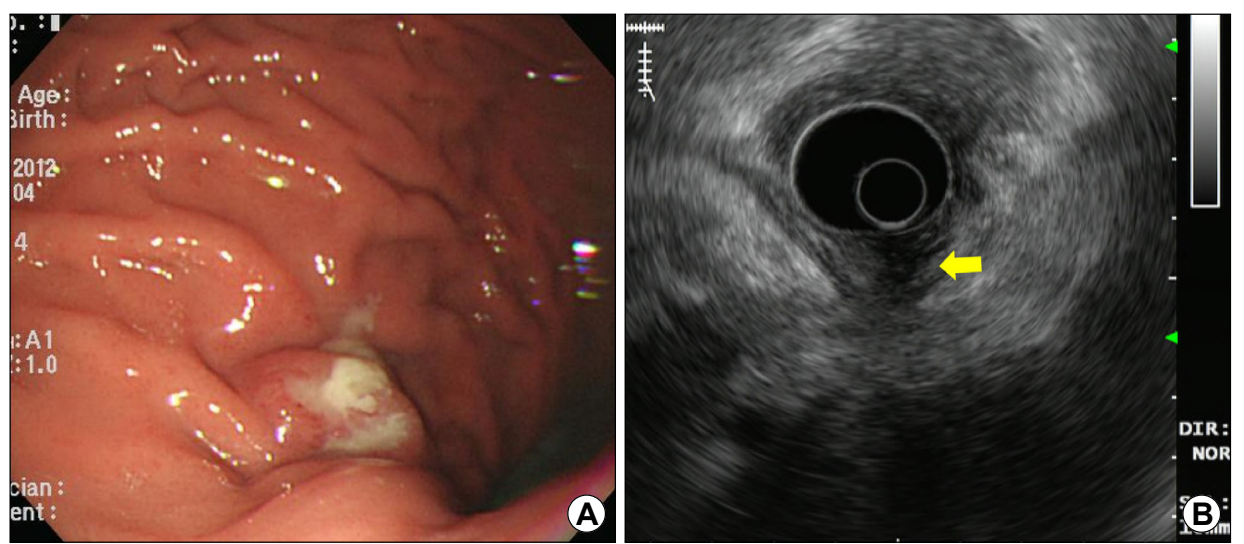

Fig. 3. (A) Follow-up endoscopy shows a subepithelial tumor-like lesion with central ulceration at the posterior wall of the upper body of the stomach. (B) EUS shows that the lesion is mainly located in the submucosal layer of the stomach without conglutination to the pancreatic mass (arrow).

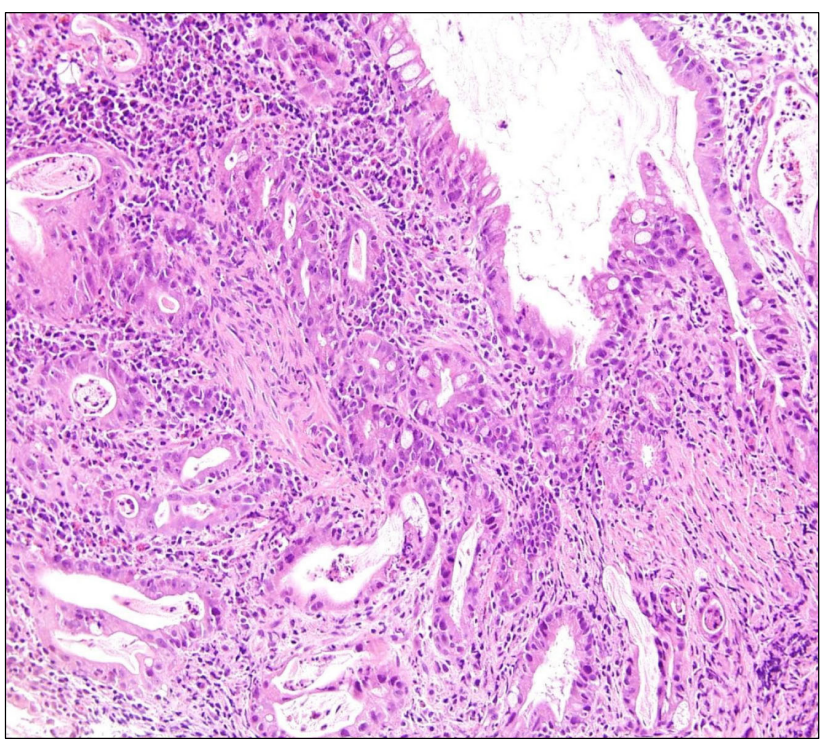

Fig. 4. The endoscopic biopsy specimen obtained from the subepithelial tumor-like lesion indicates adenocarcinoma, pathologically identical to that of primary pancreatic cancer $(\mathrm{H} \& \mathrm{E}, \times 200)$.

dality for pancreatic cancer; the adverse event rates are very low $(<1 \%$ in large centers) and the risks of tumor seeding are lower compared to transcutaneous methods. ${ }^{1}$ However, as the number of EUS-FNA increases, the possibility of needle tract seeding occurring after EUS-FNA should always be minded. ${ }^{2}$ Especially, in cases when surgical resection does not include the needle tract, such as cancers in the body or tail of the pancreas, EUS-FNA should be cautiously considered and the number of needle passes should be minimized. Close periodical exami- nations including gastroscopy should be scheduled for patients who undergo EUS-FNA without needle tract resection. ${ }^{3}$ In summary, even though needle tract seeding is a rare adverse event of EUS-FNA in pancreatic cancer, it can occur, especially when the cancer is located in the body or tail of the pancreas.

\section{CONFLICT OF INTEREST}

No potential conflict of interest relevant to this article was reported.

\section{ORCID}

Gwang Ha Kim (D) https://orcid.org/0000-0001-9721-5734

\section{REFERENCES}

1. Micames C, Jowell PS, White R, et al. Lower frequency of peritoneal carcinomatosis in patients with pancreatic cancer diagnosed by EUS-guided FNA vs. percutaneous FNA. Gastrointest Endosc 2003;58:690-695.

2. Kita E, Yamaguchi T, Sudo K. A case of needle tract seeding after EUS-guided FNA in pancreatic cancer, detected by serial positron emission tomography/CT. Gastrointest Endosc 2016;84: 869-870.

3. Tomonari A, Katanuma A, Matsumori T, et al. Resected tumor seeding in stomach wall due to endoscopic ultrasonographyguided fine needle aspiration of pancreatic adenocarcinoma. World J Gastroenterol 2015;21:8458-8461. 